Published in Proceedings of the 2018 GIoTS, Bilbao, Spain, 04-07 June 2018, whish should be cited to refer to this work. DOI: $10.1109 /$ GIOTS.2018.8534558

\title{
Improved Machine Learning Methodology for High Precision Agriculture
}

\author{
Jérôme Treboux \\ and Dominique Genoud \\ Institute of Information Systems \\ University of Applied Sciences, HES-SO Valais \\ Sierre, Switzerland \\ Email: (jerome.treboux, dominique.genoud)@hevs.ch
}

\begin{abstract}
This paper presents the impact of machine learning in precision agriculture. State-of-the-art image recognition is applied to a dataset composed of high precision aerial pictures of vineyards. The study presents a comparison of an innovative machine learning methodology compared to a baseline used classically on vineyard and agricultural objects. The baseline uses color analysis and can discriminate interesting objects with an accuracy of $(89.6 \%)$. The machine learning, an innovative approach for this type of use case, demonstrates that the results can be improved to obtain $94.27 \%$ of accuracy. Machine Learning used to enrich and improve the detection of precise agricultural objects is also discussed in this study and opens new perspectives for the future of high precision agriculture.
\end{abstract}

Keywords-Machine Learning; Prediction; Hyper-spectral images; Agricultural health; Precision agriculture; Image recognition

\section{INTRODUCTION}

Nowadays, precision agriculture aims at increasing the productivity and maximizing the yields of a crop. It can benefit the entire crop cycle through an application of the correct amount of inputs (such as water, fertilizers, pesticides or fungicides) at the exact time and the right place, or by detecting diseases in plants [1].

Because of complex landscapes or difficult topographies, the precision agriculture has not been applied to vineyards or to complex pattern fields. Currently, it is mainly used to yield monitoring or remote sensing.

New services and software are currently under development to provide better vineyards and other cultures management. For example, an automatic collection of data related to a culture, for automatic spotting, designation of zones, and prediction of the evolution of a zone [1].

Cartographic data are currently used to detect specific and characterized natural zone, especially through satellite images [2][3].

Based on these needs, the current research focuses on the detection of the vine and potential disease on infrared images taken by Unmanned Aerial Vehicle (UAV). A new flight plan for the drone is generated thanks to the automatic detection. This drone can fly to a specific region of the field, carrying and spreading out the treatment products.
Machine learning algorithms are currently widely used for other research purposes in the image recognition field. These research are focusing on satellite image mapping [4] for example or online images matching. To improve the overall accuracy and to set up a better precision for the drone route, these machine learning algorithms are applied to the pictures taken by the drone.

This article provides a state of the art in the second section. The experiment dataset is then explained, followed by the baseline description. The results are presented in the fifth section. Finally, the conclusion is detailed.

\section{STATE OF THE ART}

Image analysis is part of the machine learning field and is broadly used for research purpose as well for product usage, as described above. Multiple projects are currently running or were achieved to improve the quality of object classification. In this section, we will review the main ones.

\section{A. Pixel-based and Object-oriented classification}

Image classification is one of the most basic operations of digital image processing [5]. A widely process used is the pixel-based classification (PBC) and the object-oriented classification (OOC).

The PBC is based on conventional statistical techniques. It categorizes all the pixel into thematic classes using specific features such as spectral information. For each pixel, a spectral signature is estimated by the relative reflectance in different wavelength bands.

The OOC is not anymore classifying each pixel but objects present on the picture. It is based, for example, on spatial-spectral characteristics of the high-resolution (HR) satellite data information. The last decade's development for the OOC was mainly based on the rule-based classifier and the nearest neighbor (NN) classifier. The OOC is now broadly used and replace the PBC slowly.

The power of the $\mathrm{PBC}$ is the combined spectral response from all training pixels. Therefore, the classification method ignores the impact of mixed pixels [6]. The PBC cannot use the relationship between pixels or be applied to hyperspatial data [7]. Furthermore, the PBC cannot recognize objects much bigger than a pixel [8]. 
The OOC advantages are the use of spectral information like land types, shapes, texture parameters and relationships between contexts. The inclusion of the vector-based GIS environments improves the power of the method. And the accuracy is improved by the usage of a large set of features like the spatial, textural and contextual properties [9][10][11].

\section{B. Object-based image analysis through nonlinear scale- space filtering}

The second methodology for image analysis and object classification is the analysis through nonlinear scale-space filtering. Tzotsos, Karantzalos and Argialas [12] provide a framework to compute images at different scales and connected to a kernel-based learning machine for the classification of various earth-observations.

Creating this framework demonstrates that the multilevel object representation and support vector machine classifier possesses the desired qualitative properties. This lets the possibility to eliminate the need for tuning several parameters during the segmentation. The current algorithms outperform the previous development regarding the representation and the classification results. The Figure 1 demonstrates the accuracy for objects detection in multispectral aerial images.
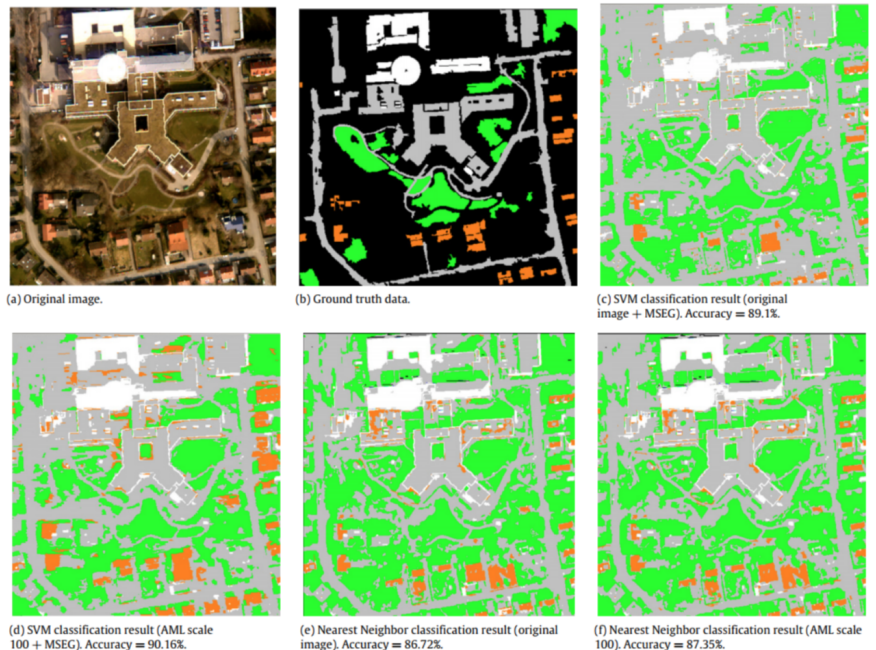

Figure 1. Classification results with and without AML filtering. Green: vegetation, grey: asphalt, orange: tile roofs, white: bright roofs.

\section{Vineyard detection and Vine discrimination from very $H R$ Satellite Data}

The newspaper Remote Sensing published a research close to the present work. The project of Karakizi, Oikonomou and Karantzalos [3] focus on the implementation of automated and efficient agriculture methods. The framework presented is based on multispectral data and proposes an object-based classification.

Multiple studies about vegetation, forest mapping, and biomass estimation are already available
$[13][14][15][16][17]$. For the vineyard detection, the number of products and studies are reduced. The difficulty in a vineyard is the leaves and trunks mixed with metal and other material to maintain them. Furthermore, they are traveled by paths and surrounded by roads. The experiment is based on satellite images of vineyards in Greece, from 2012 to 2013. The images format is close to the present use case as it is GeoTIFF format.

The paper presents a vine variety discrimination. This supervised learning and majority vote method increase the overall accuracy close to $92 \%$. Indeed, even in a same part of the vineyard, multiple varieties are detected. This misclassification can come from disease or mistakes done by the ground truth definition.

\section{DATASET}

The dataset is composed of images from 5 vineyards in Valais, Switzerland taken by a drone. The file format to keep the geo information is GeoTIFF, created with the software Pix4D. Images are handled through a GIS software or a Python script to extract the relevant information (Bioformats for example) for further classification.

For the baseline, using the work of Lorenzo [18], the images are not manipulated and used as-is for the experiment. The first analysis is made to understand the images format and is described in the next section.

For the improvement of the object detection experiment, the dataset was transformed to meet some requirements of the machine learning algorithm. For the present work, a division of the images in smaller tiles of 30x33 pixels is done. Each tile contains mainly one of the three categories: (1) Road, (2) Vineyard or (3) Other (See Figure 2). Multiple experiments were run to calculate the best size of the tiles to meet two primary criteria: being large enough to be classifiable and not too large to contain multiple classes. This represents 13'005 images for the full dataset. Each image is manually labeled. The summary of this classification is described in the Table I.

The dataset is divided into two main partitions for training and testing. To obtain the most accurate results, the train set is composed of $90 \%$ of the dataset taken with a balanced selection, and the remaining $10 \%$ stay for the testing process.

Table I

FULL DATASET SUMMARY OF DRONE IMAGES AFTER SUBDIVISION INTO TILES. EACH CATEGORY CONTAINS THE NUMBER OF TILES MANUALLY LABELED. SOURCE: PREVIOUS PROJECT RUNNING ON DEEP LEARNING AND MACHINE LEARNING COMPARISON.

\begin{tabular}{|c|c|c|}
\hline Class & Number of tiles & Size of tiles \\
\hline vineyard & $7^{\prime} 022$ & $30 \times 33$ pixels \\
\hline road & $2{ }^{\prime} 082$ & $30 \times 33$ pixels \\
\hline other & $3^{\prime} 903$ & $30 \times 33$ pixels \\
\hline Number of tiles per class & \multicolumn{2}{|c|}{$13^{\prime} 005$} \\
\hline Total number of tiles & \multicolumn{2}{|c|}{$2^{\prime} 601$} \\
\hline
\end{tabular}



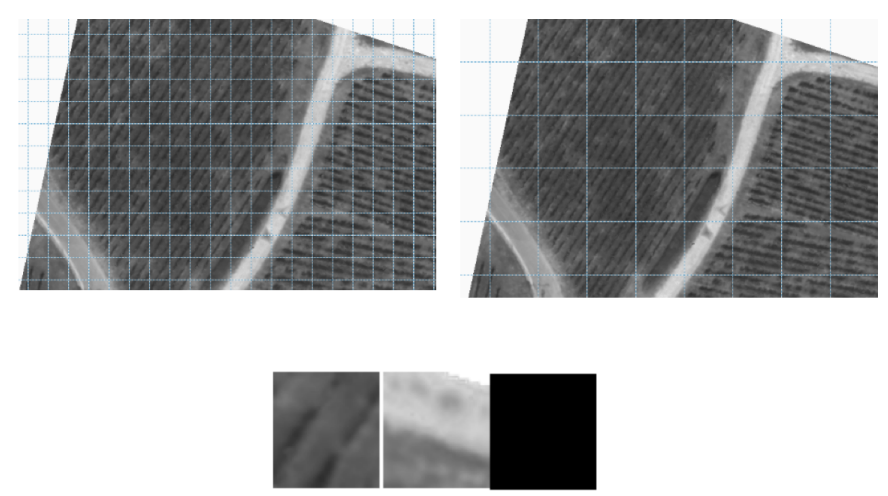

Figure 2. Vineyard split with tiles of 30x33px (left) and 78x80px (right). On the left, some objects are on the same tile, but rarely a vineyard and other. On the right, objects are mixed.

\section{BASELINE DESCRIPTION}

This part is based on multiple framework and studies, combined to obtain the best ground truth. The basis is taken from the research of Lorenzo Comba \& al. [18]. They used mainly the image intensity and Hough Transform to categorize vineyard.

The first comparison of two specific objects is provided. It means that two zones, road and vineyard, are extracted to evaluate the color intensity. On Figure 3, the original vineyard is presented with the 2 zones selected.

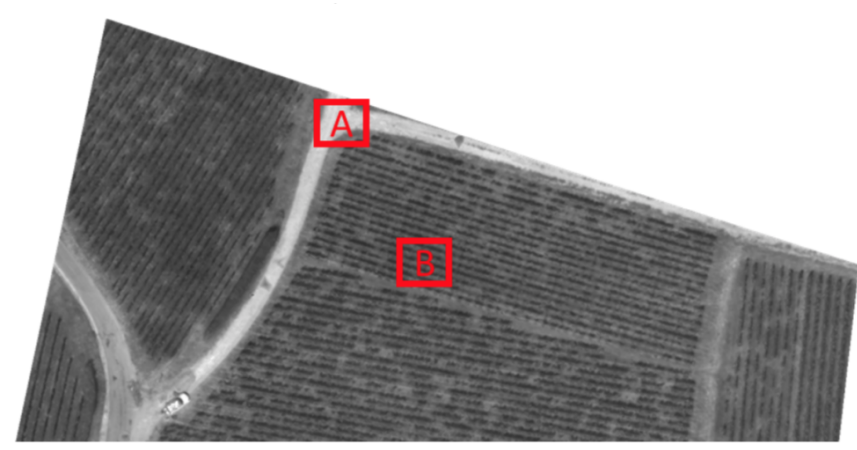

Figure 3. Original vineyard image. (A) is the zone presenting a road and (B) is the reference zone for vineyard

The difference of the intensity between the classes road and vineyard is presented on the Figure 4. The color intensity is stronger on the vineyards compared to the road. This confirms the potential usage of intensity as a feature for the development of the algorithm.

To increase the accuracy, morphological processes are applied. Two morphological transformations are applied: the opening and the closing transformations. The opening process removes noise with a succession of erosion and dilation process. Then the closing process applies dilation and erosion to close small holes inside the foreground objects (OpenCV). This process consolidates the foreground

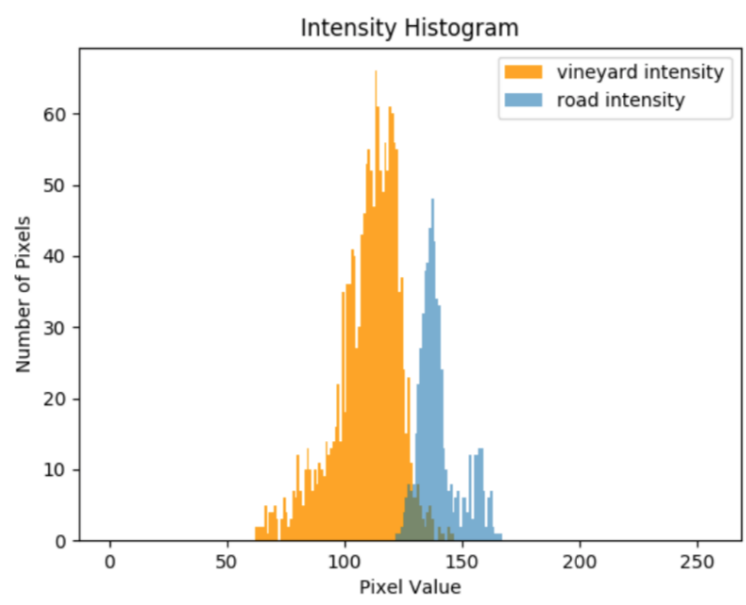

Figure 4. The Intensity Histogram shows the difference between the two classes, road and vineyard. It shows the number of pixel per intensity for each class

part, in this case, the vineyards (See Figure 5). The result shows the different zones present in the picture. There are some issues with the road (bottom left of the graph) that is associated with a vineyard because of the similar color intensity.
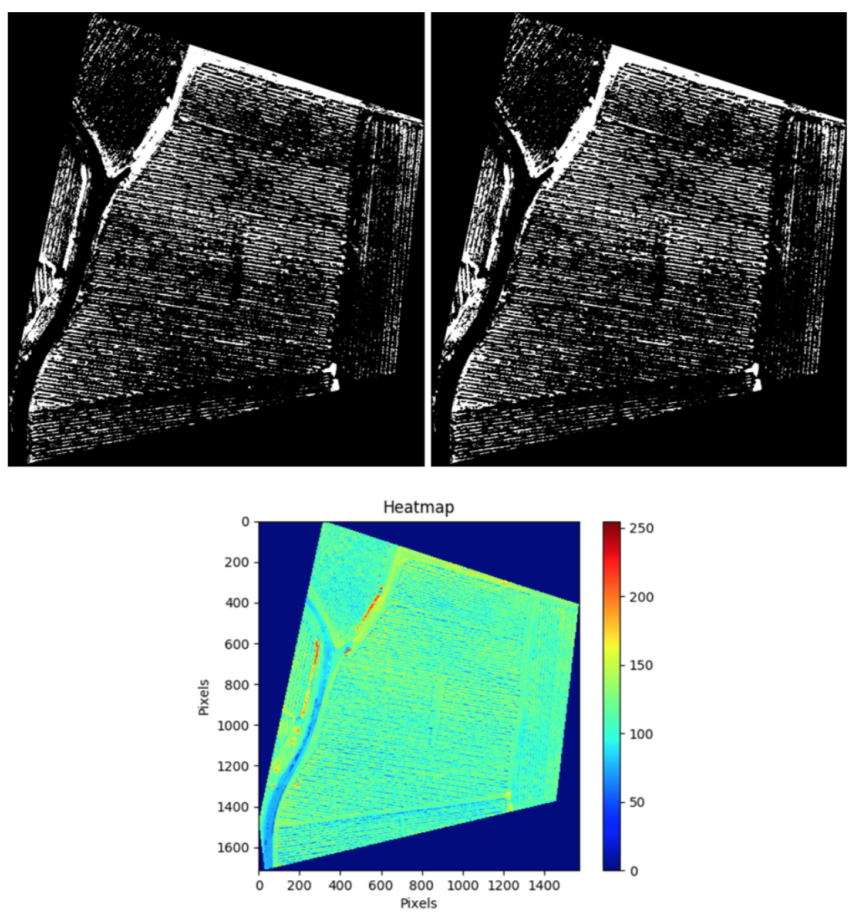

Figure 5. Increased intensity and reduced noise on the transformed image (top left) compared to the original black and white image (top right). The heat map of intensity shows the vineyard detection in blue (bottom). 


\section{Machine LEARNing IMPROVEMENT With DTE}

The Decision Tree Ensemble (DTE) is based on features extracted from each tile of the vineyard. In a way to keep the stability and consistency of the algorithm the extraction process is applied at the beginning. Three groups of features are extracted from each tile. It represents 86 features in total, summarized below:

- First order statistics: Min, max, mean, geometric mean, sum, variance, skewness, kurtosis, etc.

- Tamura: Granularity, Contrast, Kurtosis of directionality, Standard Deviation Directionality, Max Directionality and Skewness.

- Haralick: Statistical features based on gray-level cooccurrence matrix.

A backward elimination process is applied to the algorithm to reduce the number of features and increase the overall accuracy by removing noisy features. Duplicate features are removed and as well unrelated features for a black and white image. Following the elimination process, the smallest and most powerful combination for this case uses only 16 features. The error is reduced to 0.047 .

Based on our iterative experiments, algorithm-fine tuning is done over similar dataset sampling. The final configuration of the Tree Ensemble Learner is based on the following criteria:

- Split criterion: Information Gain Ratio

- Tree depth: No limitation

- Minimum child node size: No minimum value

- Number of trees: 100

- Rows data sampling method: Stratified

- Attributes data sampling method: Square root

\section{RESULTS}

The results are obtained after a specific tuning for each algorithm and process. A detailed analysis of the baseline and the DTE is presented below. It shows the difference regarding accuracy, as well as the standard error generated.

\section{A. Baseline}

The baseline shows an excellent accuracy in detecting the vineyards within an aerial image. Moreover, the development is stable and not too difficult to diversify to detect other viticulture types. The overall detection accuracy of the vines is a bit lower than presented in [18]. A comparative table is presented below, showing the stability of the process between the study image [18] and our local images (See Table II).

\section{B. Decision Tree Ensemble}

The final trained Decision Tree Ensemble model is applied to each training set tile. A strict process is applied to have a comparison point at the end of the research, and it is based on the following steps:
Table II

COMPARATIVE RESULTS TABLE WITH THE PREVIOUS STUDY AND LOCAL VINEYARDS APPLICATION

\begin{tabular}{|c|c|c|c|}
\hline & $\begin{array}{c}\text { Best detection } \\
\text { within the study }\end{array}$ & $\begin{array}{c}\text { Local } \\
\text { vineyard 1 }\end{array}$ & $\begin{array}{c}\text { Local } \\
\text { vineyard 2 }\end{array}$ \\
\hline Accuracy & $96.06 \%$ & $90.02 \%$ & $89.6 \%$ \\
Std err & N/D & $\pm 1.17 \%$ & $\pm 1.01 \%$ \\
\hline
\end{tabular}

- Image metadata extraction: Image reader, image labeling and features extraction

- Feature elimination: Based on the model generated during the training process to keep and delete the same columns

- Prediction: Applying the model created before on the test set

- Evaluation: Generating a scorer to understand the performance of the classification

The final overall accuracy of the algorithm is of $94.275 \%$ with a standard error of $\pm 1.14 \%$ (See Table III). The vines are very well classified. Few of them are wrongly classified. The DTE is very stable throughout all iterations and provides a stable accuracy. The misclassification is due to the presence of mixed objects on the same portion of the image.

To better understand the potential sources of error and be able to correct the classification algorithm, a manual exploration of the resulting dataset is necessary.

Table III

THIS TABLE PRESENTS THE CONFUSION MATRIX OF THE DECISION TREE ENSEMBLE CLASSIFICATION WITH THE OVERALL RESULT AND STANDARD ERROR.

\begin{tabular}{|c|c|c|c|}
\hline Class / Prediction & vineyard & road & other \\
\hline vineyard & 136 & 7 & 0 \\
\hline road & 8 & 36 & 0 \\
\hline other & 0 & 0 & 75 \\
\hline Accuracy & $94.275 \%$ \\
\hline Std error & $\pm 1.14 \%$ \\
\hline
\end{tabular}

\section{CONCLUSION}

However, the process is not functional when the vineyards are not aligned. Indeed, in some region (especially north of Italy) a lot of vineyards are over wood construction and not anymore aligned. An adaptation of the process should be done to detect this new type of agriculture. This process can be improved with more powerful development and computers to reduce the computing time. It is a very interesting basis for the research work and an accurate benchmark to compare the current results. A good point for this study is the time winning without jumping in a machine learning process. Indeed, image processing is necessary but no need of image splitting process and labeled data. 


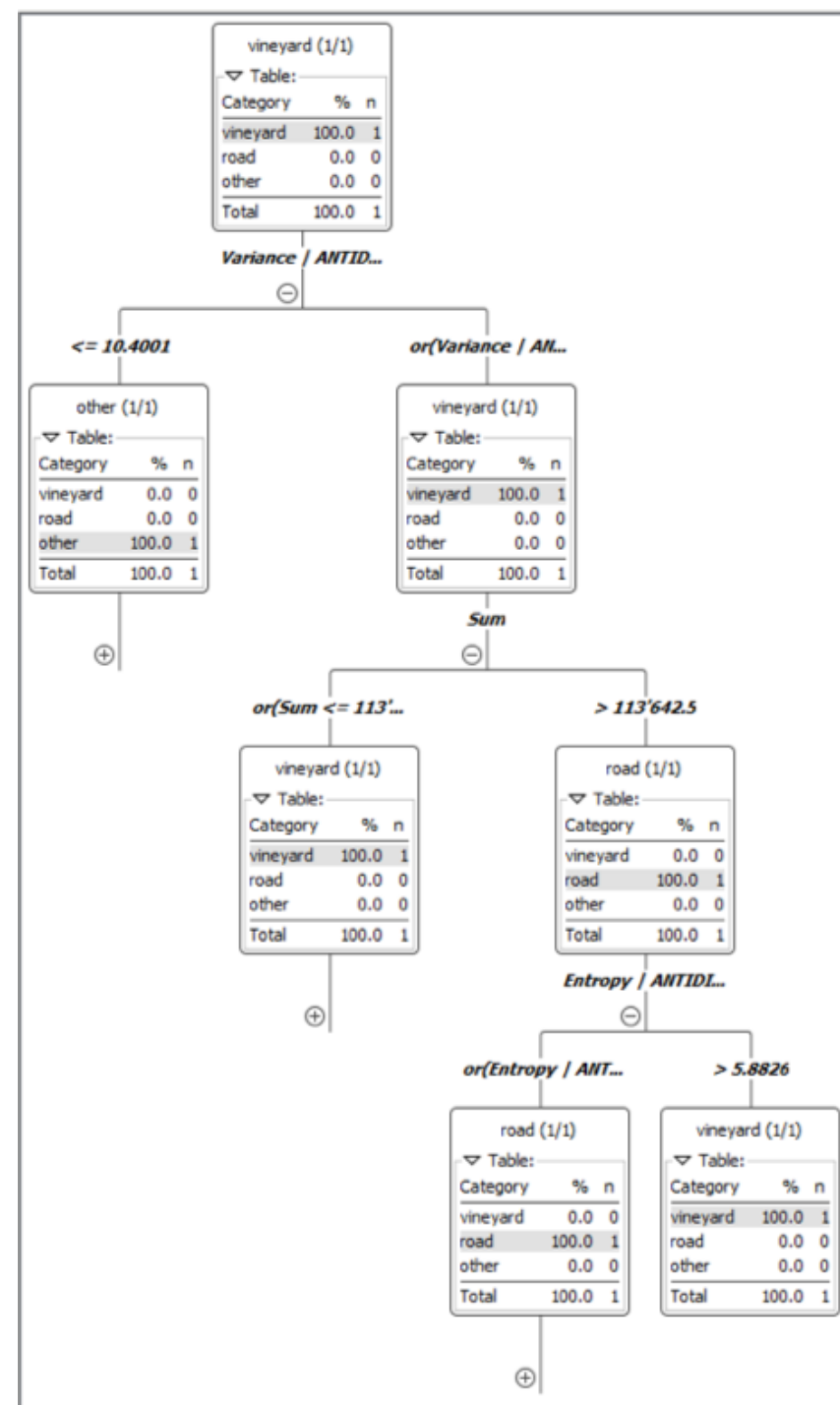

Figure 6. Overvieew of the structure of the Decision Tree Ensemble model created on the dataset presented above. This extract of the structure shows that the first feature to separate the dataset is the VARIANCE / ANTIDIAGONAL which was selected during the features selection process.

\section{ACKNOWLEDGMENT}

The authors would like to highlight the successful collaboration with the company AgroFly, based in Switzerland, for the images provided and the use case definition. A special thank you to the Institute of Business Information of the University of Applied Sciences of Western Switzerland for the support and the tools furnished.

\section{REFERENCES}

[1] AgroFly SA, High Precision Forecast with Agro Drones (UAV), SPSS, Sierre, Switzerland, 2016.

[2] G. Popkin, Satellite alerts track deforestation in real time, nature.com, 530(7591), 392-393, 2016.
[3] C. Karakizi, M. Oikonomou, and K. Karantzalos, Vineyard Detection and Vine Variety Discrimination from Very High Resolution Satellite Data, Remote Sens, 8, 235, 2016.

[4] B. Schultz, M. Formaggio, A. R. Del' Arco Sanchez, L. Barreto Luiz and C.N Atzberger, Self-Guided Segmentation and Classification of Multi-Temporal Landsat 8 Images for Crop Type Mapping in Southeastern Brazil, Remote-Sensing, 1448214508, 2015.

[5] S. D. Jawak, P. Devliyal and A. J. Luis, A Comprehensive Review on Pixel Oriented and Object Oriented Methods for Information Extraction from Remotely Sensed Images with a Special Emphasis on Cryospheric Applications, ScriRes, 4, 177-195, 2015.

[6] D. Lu and Q. Weng, Spectral Mixture Analysis of the Urban Landscape in Indianapolis with Landsat ETM+ Imagery, Photogrammetric Engineering and Remote Sensing, 70, 1053$1062,2004$.

[7] H. Sridharan and F. Qiu, Developing an Object Based Hyperspectral Image Classifier with a Case Study Using WorldView-2 Data, Photogrammetric Engineering and Remote Sensing, 79, 1027-1036, 2013.

[8] P. Corcoran, A. Winstanley and P. Mooney, Segmentation Performance Evaluation for Object Based Remotely Sensed Image Analysis, International Journal of Remote Sensing, 31, 617-645, 2010.

[9] Z. Chen, X. Ning and J. Zhang, Urban Land Cover Classification Based on WorldView-2 Image Data, IEEE International Symposium on Geometrics for Integrated Water Resource Management, Lanzhou, 2012.

[10] P. Aplin and G. Smith, Advances in Object Based Image Classification, The International Archives of Photogrammetry, Remote Sensing and Spacial Information Sciences, 37, 725728, 2008.

[11] D. Liu and F. Xia, Assessing Object.Based Classification: Advantages and Limitations, Remote Sensing Letters, 1, 187194, 2010.

[12] A. Tzotsos, K. Karantzalos and D. Argialas, Object-based image analysis through nonlinear scale-space filtering, Journal of Photogrammetry and Remote Sensing, 66, 2-16, 2011.

[13] S. Rapinel, B. Clément, S. Magnanon, V. Sellin and L. Hubert-Moy, Identification and mapping of natural vegetation on a coastal site using WorldView-2 satellite image, J. Environ. Manag., 144, 236-246, 2014.

[14] R. Lottering and O. Mutanga, Optimizing the spatial resolution of WorldView-2 imagery for discriminating forest vegetation at subspecies level in KwaZulu-Natal, South Africa, Geocarto Int, 2015.

[15] M. Immitzer, C. Atzberger and T. Koukal, Tree Species Classification with Random Forest Using Very High Spatial Resolution 8-ban WorldView-2 Satellite Data, Remote Sens., 4, 2661-2693, 2012. 
[16] R. Pu and S. Landry, A comparative analysis of high spatial resolution IKONOS and WorldView-2 imagery for mapping urban tree species, Remote Sens., 124, 516-533, 2012.

[17] O. Mutango, E. Adam and M. Cho, High density biomass estimation for wetland vegetation using WorlView-2 imagery and random forest regression algorithm, Int. J. Appl. Earth Obs. Geoinform., 18, 399- 406, 2012.

[18] L. Comba, P. Gay, J. Primicerio and D. R. Aimonino, Vineyard detection from unmanned aerial systems images, Computers and Electronics in Agriculture, 114, 78-87, 2015. 